\title{
Seasonal distribution and phylogenetic analysis of human metapneumovirus in children with acute respiratory tract infections admitted to a general hospital in Sri Lanka
}

\author{
MVM Divarathna ${ }^{1}$, RAM Rafeek ${ }^{1}$, AJ Morel $^{2}$, S Jagoda ${ }^{3}$, F Noordeen ${ }^{1}$
}

Introduction and Objectives:Human metapneumovirus (hMPV) is one of the major causes of acute respiratory tract infections (ARTI) in childrenworldwide. hMPV has two major types, A and B and four subtypes, A1, A2, B1, and B2.hMPV A2 is further divided into three sub-genotypes, A2a, A2b and A2c. This study was undertaken to determine the distribution, seasonality and subtypes of hMPV circulating in children $<5$ years with ARTI.

Methods:A total of 500 nasopharyngeal aspirates were collected from children $<5$ years hospitalized at the District General Hospital, Kegalle between May 2016 and July 2018. hMPV was detected by a conventional reverse transcription PCR (RT-PCR). Of the 119 hMPV positive children's PCR products, 17 randomly selected samples were characterized using direct Sanger sequencing ( $\mathrm{n}=15)$ and Thymine and Adenine (TA) cloning followed by Sanger sequencing $(\mathrm{n}=2)$. The sample sequencing was limited to 17 due to funding restraints.

Results: hMPV was detected with a prevalence of 23.8\% (119/500) inchildren < 5 years of age throughout the study period with two major peaks occurring in February to May in 2017 and May to July in 2018 and two minor peaks occurring in July 2017 and February 2018. The sequenced hMPV strains belonged to type A and the subtype A2. It is not clear whether the $\mathrm{A} 2$ strains belong to the sub-genotypes A2b or A2c

Conclusions:hMPV infection had a prevalence of $~ 24 \%$ and is considered high compared to its prevalence in some other countries. The infection was distributed throughout the year in the study area. The sequenced 17 hMPV samples were hMPV A2 genotype.

Key words: Human metapneumovirus, Acute respiratory tract infections, Children, Phylogeny

Funding: National Science Foundation of Sri Lanka (NSF/SCH/2017/01).

\footnotetext{
${ }^{1}$ Department of Microbiology, Faculty of Medicine, University of Peradeniya, Sri Lanka

${ }^{2}$ District General Hospital, Kegalle, Sri Lanka

${ }^{3}$ Department of Veterinary Pathobiology, Faculty of Veterinary Medicine \& Animal Science, University of Peradeniya, Sri Lanka
} 